\title{
Les femmes kanak sont fatiguées de la violence des hommes
}

Christine Salomon et Christine Hamelin

\section{(2) OpenEdition \\ 12 Journals}

Édition électronique

URL : http://journals.openedition.org/jso/1009

DOI : $10.4000 /$ jso. 1009

ISSN : $1760-7256$

Éditeur

Société des océanistes

Édition imprimée

Date de publication : 1 décembre 2007

Pagination : 283-294

ISBN : 978-2-85430-010-9

ISSN : 0300-953x

Référence électronique

Christine Salomon et Christine Hamelin, «Les femmes kanak sont fatiguées de la violence des hommes ", Journal de la Société des Océanistes [En ligne], 125 | Année 2007-2, mis en ligne le 01 décembre 2010, consulté le 21 avril 2019. URL : http://journals.openedition.org/jso/1009 ; DOI : 10.4000/jso.1009

\section{(c) Tous droits réservés}




\title{
Les femmes kanak sont fatiguées de la violence des hommes ${ }^{1}$
}

\author{
par
}

\author{
Christine SALOMON* et Christine HAMELIN ${ }^{* *}$
}

\section{RÉSUMÉ :}

Le rééquilibrage économique entrepris dans les années 1990 pour combler le fossé social qui séparait en Nouvelle-Calédonie les Kanak des autres communautés, l'engagement des femmes kanak dans le mouvement indépendantiste et, plus récemment, l'application de la parité politique, ont provoqué des mutations importantes dans la vie matérielle des femmes mais aussi dans leurs représentations du genre. Nos enquêtes ethnographiques et les résultats d'une étude par questionnaire en population générale féminine que nous avons menée sur le thème des violences faites aux femmes indiquent qu'aujourd'hui une majorité de femmes kanak ne légitiment ni les viols ni les coups, y compris quand l'auteur est le conjoint. Elles contestent ainsi un ressort de l'ordre masculin et mettent en question les constructions anciennes de la conjugalité. Alors que les violences constituaient jusqu'il y a peu un moyen généralisé de la domination masculine, leur mise en cause idéologique, massive chez les plus jeunes des Kanak et chez celles qui résident en ville, témoigne à l'échelle des individus d'ouvertures nouvelles et plus généralement d'une renégociation des rapports sociaux de sexe que nous essayons ici de mieux comprendre et d'inscrire dans l'histoire politique et sociale récente du pays.

Mots-CLÉs : genre, violences, femmes kanak, changement social, politique.

\begin{abstract}
:
The engagement of Kanak women with the proIndependence movement, the economic rebalancing programme launched in the 1990's to bridge the social gap in New Caledonia between the Kanak and the other communities, and more recently the application of political parity have led to deep change in women's everyday lives but also in their gender representations. Our ethnographic investigation and the results of our questionnaire survey on violence among the general female population show that, today, a majority of Kanak women no longer legitimise rape or physical violence even if the perpetrator is the partner. In this way they are challenging one of the foundations of the male order and querying long-standing constructions of the conjugal relationship. Violence was until recently a generalised way of imposing male domination. Its ideological challenging, now on a massive scale by the youngest Kanak women and urban Kanak women, shows that new openings are emerging at the individual level and that, more generally, there is a renegotiation of gender social relationships that we will try better understanding here while setting it into the background of the country's recent political and social history.
\end{abstract}

KEYwORDS: gender, violence, Kanak women, social change, politics.

You can't be neutral on a moving train

(Howard Zinn)

1. Ce titre fait allusion au propos de l'hérö̈ne de L'Épave, le roman de Déwé Gorodé : «je t'attendrai ici, au paradis des femmes, quand tu seras fatiguée de la violence des hommes ».

* INSERM u 687/IFr 69 St-Maurice, Christine.Salomon@st-maurice.inserm.fr

** INSERM U 687/IFR 69 St-Maurice, Christine.Hamelin@st-maurice.inserm.fr 
L'ethnologie des sociétés kanak s'est caractérisée depuis ses débuts par une certaine tradition de dénonciation des violences tant politiques les guerres coloniales et les spoliations foncières - que symboliques et structurelles - le racisme institutionnalisé, l'ancrage ethnique des inégalités sociales - sans que les chercheurs qui se sont succédé depuis Maurice Leenhardt en NouvelleCalédonie aient été pour autant accusés de déroger à la distance critique, ni trop près ni trop loin, particulièrement valorisée dans le champ académique français. En revanche elle est restée longtemps muette sur les violences faites aux femmes. Faire de ces violences un objet de recherche passait jusqu'il y a peu pour une posture militante féministe sujette à réprobation. L'ethnographe était soupçonnée de générer un climat non scientifique de dénonciation, avec des propos ou un ton ethnocentriques, susceptibles de renforcer le discrédit colonial à l'égard de la culture kanak. Ou alors de généraliser abusivement telle situation ou vécu particuliers, ce qui est une façon de nier la fréquence des faits et leur légitimation sociale. Ces critiques ne sont pas sans rappeler celles portées, dans le débat ouvert par Current Anthropology, par les tenants de la neutralité et de l'objectivité scientifiques aux partisans du primat de l'éthique et d'une anthropologie militante, censés créer un climat de dénonciation et de rage - «it creates a climate of denunciation and rage»-, et relever de l'ethnocentrisme - «finally, the current model is ethnocentric» (D'Andrade, 1995 : 407-408).

Dans le monde kanak, les violences faites aux femmes ont pourtant la particularité d'être tout à fait communes, de déborder l'intimité familiale et conjugale pour se manifester aussi en public, ce qui les rend directement observables du moins pour les agressions verbales et physiques, les agressions sexuelles étant quant à elles facilement accessibles au travers des récits et des témoignages. Il est certainement inconfortable pour l'ethnographe de se confronter à « la prévalence et l'aspect normal du viol » et difficile d'en rendre compte sans stigmatiser davantage le groupe social étudié lorsqu'il est lui-même dominé (Bourgois, 2001 : 243). Mais tout dévoilement serait-il voué à contribuer à une sorte de «pornographie de la violence» et faudrait-il, de peur de "donner des armes au racisme » (Bourdieu, 1998 : 122), de « renforcer la perception négative aux yeux de lecteurs mal intentionnés » (Bourgois, 2002 : 116), s'empêcher d'analyser précisément un instrument aussi massif de domination et se cantonner à la dimension proprement symbolique de celle-ci?

Lors de notre arrivée en Nouvelle-Calédonie, en 1989 pour Christine Salomon et en 1992 pour Christine Hamelin, le pays - qui était encore un territoire d'Outre-Mer - sortait à peine de plusieurs années de violences politiques ( les événements »). Notre engagement dans la solidarité anti-coloniale, aux côtés de la plupart de nos collègues, nous prédisposait à une vision positive, si ce n'est idéalisée, des sociétés kanak. Étudiant pour l'une les représentations du corps et de la maladie, pour l'autre le phénomène d'urbanisation, nous n'avions pas envisagé de centrer notre travail sur les violences de genre. Pourtant, leur prégnance dans la vie sociale et l'impossibilité de les passer sous silence nous apparurent assez rapidement. Dans des rassemblements (à l'occasion d'un mariage, d'une réunion politique), dans la rue, dans les familles dont nous partagions le quotidien, il nous arriva d'assister à des scènes de violences conjugales. Les faits étaient considérés comme ordinaires, aucun tiers ne protestait ouvertement ni n'intervenait et l'on nous fit comprendre qu'il n'était pas question de s'interposer. Nous vîmes aussi des femmes que nous connaissions soudain réapparaître avec une blessure provoquée par un coup de sabre d'abattis ou un bras dans le plâtre et, en réponse aux questions sur ce qui leur était arrivé, mentionner sans le cacher mais sans s'étendre, que c'était dû à leur conjoint: "Ça, c'est le travail à mon vieux $»$.

De plus, dans cette première moitié des années 1990, les procès des crimes sexuels, notamment des viols collectifs, faisaient régulièrement les gros titres de la presse locale et alimentaient les commentaires sur l'actualité. Il n'y avait pas encore chez les auteurs l'autocensure qui devait apparaître ensuite avec leur lourde pénalisation par le tribunal : des hommes adultes, dans des conversations avec Christine Salomon à propos des affaires judiciaires, en parlaient facilement comme d'une initiation à la sexualité, une activité adolescente masculine ordinaire. De leur côté, toutes les femmes connaissaient des filles de leur entourage qui avaient été victimes de tels viols. Certaines témoignaient même directement d'agressions ${ }^{2}$. Il nous sembla vite évident que les rapports de genre étaient essentiels à analyser dans la structuration des sociétés kanak et qu'on ne pouvait aborder l'ordre masculin uniquement au travers du dispositif symbolique qui le sous-tend, en éliminant du champ de description et de réflexion, les violences physiques et sexuelles. 


\section{L'apport de la statistique}

Encouragées par l'effet de dévoilement des premiers résultats de l'enquête nationale sur les violences envers les femmes en France (ENVEFF), nous fîmes le projet d'une étude par questionnaire en population générale féminine sur les violences en Nouvelle-Calédonie. Ce travail quantitatif pouvait sembler peu satisfaisant intellectuellement à certains ethnologues, mais établir la mesure du phénomène nous paraissait nécessaire et, comme Nancy Scheper-Hugues, nous pensions « que, dans de tels cas, ce que les anthropologues peuvent faire de mieux est un empirique et humble travail de recensement » $(1996: 891)^{3}$. Le projet reçut très tôt le soutien de Déwé Gorodé ${ }^{4}$ et, grâce à elle, celui du gouvernement de la Nouvelle-Calédonie, ainsi que celui des deux provinces gérées par les indépendantistes, le Nord et les Îles. La contestation des violences engagée par les associations de femmes dans le pays que nous relatons plus loin favorisa l'accueil réservé sur place à cette étude.

L'enquête s'est déroulée en 2002-2003, auprès de 1012 femmes de 18 à 54 ans, interrogées en face à face parmi lesquelles $441 \mathrm{Kanak}^{5}$. Pour des raisons de confidentialité, elle était présentée localement comme une enquête sur la santé et les conditions de vie des femmes. Après un module général, les répondantes étaient interrogées sur une série de faits qui avaient pu se produire dans leurs différents cadres de vie au cours des douze derniers mois. Le questionnaire se terminait par un module qui renseignait sur les violences graves subies antérieurement, notamment les violences sexuelles.

L'analyse des données confirme la fréquence des violences subies par les femmes et montre le lien statistique fort entre ces violences et la faiblesse de leurs ressources économiques personnelles. Dans la mesure où, en NouvelleCalédonie aujourd'hui, inégalités socio-économiques et communautaires se recoupent largement, les femmes kanak, en tant que groupe, ont la situation la plus défavorable. Elles subissent ce que Paul Farmer (1997) et Nancy ScheperHugues (1996) décrivent comme une violence structurelle, qui combine des conditions de vie défavorisées, un accès moindre aux ressources tant matérielles que sociales et symboliques, et l'exposition aux violences de genre. Au cours de l'année précédant l'enquête, $34 \%$ d'entre elles ont vécu une ou des agressions physiques et $17 \%$ une ou des violences sexuelles graves (tentatives de viol ou viol). C'est dans la sphère conjugale que ces violences sont les plus fréquentes, mais il existe aussi une proportion non négligeable de violences physiques $(10 \%)$ et sexuelles $(3 \%)$ commises sur les femmes adultes dans la famille par un parent autre que le conjoint (Hamelin et Salomon, 2004). En outre, dans les espaces publics, $3 \%$ de femmes kanak déclarent des agressions physiques et $4 \%$ des tentatives de viol et des viols.

Les résultats permettent aussi d'évaluer précisément la proportion des recours médicaux, associatifs et judiciaires et montrent qu'ils ne constituent que le sommet émergé de l'iceberg, car, comme ailleurs, la plupart des victimes renoncent encore à faire valoir leurs droits, soit qu'elles ne les connaissent pas, soit qu'elles aient honte, qu'elles aient peur des représailles ou qu'elles pensent que cela ne servirait à rien. Mais bien que les recours apparaissent encore marginaux, les réponses à l'échelle d'acceptabilité des violences du questionnaire indiquent qu'une immense majorité des femmes kanak aujourd'hui désapprouve clairement les violences, y compris celles du conjoint, quelles qu'en soient les circonstances, et va jusqu'à contester les ressorts de l'ordre masculin. Alors que les violences constituaient jusqu'il y a peu un moyen généralisé de la domination masculine, leur mise en cause idéologique, massive chez les plus jeunes des Kanak et chez celles qui résident en ville (dans le Grand Nouméa), s'impose comme l'un des résultats les plus importants et les moins attendus de l'enquête. Elle corrobore la progression de l'idée d'égalité déjà soulignée dans des articles basés sur des recherches purement qualitatives (Salomon, 2000a, 2003) et témoigne d'ouvertures nouvelles que nous essayons ici de mieux comprendre et d'inscrire dans l'histoire politique et sociale récente du pays.

\section{La centralité des violences faites aux femmes}

Deux principes, le genre et l'âge, servaient dans le monde kanak ancien à naturaliser les

3. «I have suggested that in instances like these the anthropologists might best serve as a humble and empirical clerk of the records» (traduit par nos soins).

4. Déwé Gorodé, militante indépendantiste du PALIKA et écrivaine féministe, était alors ministre de la Culture et viceprésidente du premier gouvernement du pays (2001-2004). Dans le second gouvernement (depuis juin 2004), elle est en plus chargée de la Condition féminine, responsabilité qui n'existait pas auparavant.

5. Nous avons constitué un échantillon aléatoire stratifié sur l'âge à partir de la liste électorale qui, en Nouvelle-Calédonie, est une source fiable en raison du fort taux d'inscription. Seules 7,9\% des femmes contactées n'ont pas participé ( $5 \%$ de refus exprimés et $2,9 \%$ de rendez-vous non honorés). 
inégalités défavorisant les gens du commun par rapport à ceux de qualité, les lignages cadets par rapport aux aînés, les jeunes par rapport aux vieux et les femmes par rapport aux hommes. Alors que les lignages de moindre importance au terme de stratégies politiques complexes pouvaient espérer accéder à un rang supérieur, les cadets voir disparaître les aînés et les jeunes devenir vieux un jour, nous avons montré dans de précédents travaux que les femmes, au nom du biologique et de leur impureté, étaient définitivement exclues des sphères du pouvoir (Salomon, 1998, 2000b, 2002, 2003). La place qui leur était assignée était celle de procréatrice et elles se trouvaient cantonnées au rôle de gardienne du foyer. Il arrive d'ailleurs encore d'entendre dans une langue kanak du centre nord de la Grande Terre (le paicî) les désigner métaphoriquement par « feu » ou bien « marmite », et dans une autre (le a'jië) par « rien $»^{6}$, termes qui donnent une idée $\mathrm{du}$ statut social qui leur est associé. Tous les récits de femmes kanak âgées (c'est-à-dire nées vers les années 1910-1920) que nous avons recueillis évoquent la pénibilité de leurs vies et les châtiments corporels infligés à celles qui ne se soumettaient pas assez. Ainsi rechigner ouvertement aux tâches domestiques, ne pas se courber en passant devant les hommes, parler fort ou s'opposer en public à eux exposait à recevoir des coups, surtout une fois mariée. Les épouses, en effet, pouvaient être impunément frappées non seulement par leur mari et la mère de celui-ci, mais aussi par les frères réels et classificatoires $d u$ mari, ainsi que par les grands-pères de ce dernier dans les cas où l'on considérait qu'elle manquait à ses devoirs (Salomon, 2003). Des femmes aujourd'hui font encore parfois une différence entre ce qu'elles appellent en français se faire « bousculer », c'est-à-dire recevoir des gifles ${ }^{7} \mathrm{ou}$ des coups portés à mains nues, présenté comme une expression banale d'autorité masculine, et se faire « saigner » ou " damer », c'est-à-dire battre jusqu'au sang ou à la perte de connaissance, le plus souvent avec un objet, interprété comme l'effet d'un mécontentement plus sérieux ou de la méchanceté du conjoint. Dans l'enquête par questionnaire, une femme kanak en couple sur trois $(32 \%)$ a déclaré avoir subi au cours des douze derniers mois au moins une fois une violence physique perpétrée par son conjoint ${ }^{8}$.

Les règles sociales de hiérarchie et d'alliance, enseignées aux filles et aux garçons depuis le tout jeune âge, fixent les groupes sociaux au sein desquels plus tard leur partenaire devra être choisi. Autrefois, les mariages étaient décidés souvent dès l'enfance sans que les intéressés ne soient consultés. La polygynie rendait la contrainte plus violente sur les filles. « Si l'enfant, devenue jeune fille, voulait un autre jeune homme que son époux légitime de naissance, on saurait bien la mettre à la raison. Quant au jeune homme, s'il se sent de taille à soutenir la lutte et vouloir une autre femme que sa fiancée légitime, libre à lui, à condition que dans son ménage bigame ou polygame, sa vraie femme ait toujours une place» (Leenhardt, 1907 : 39). Il arrive encore, surtout aux Îles Loyauté, que les parents imposent aux jeunes de renoncer à un(e) partenaire avec qui ils ont une liaison installée et parfois un ou plusieurs enfants. I. Leblic montre également pour la région paicî (centre nord de la Grande Terre) que, dans une majorité des cas, la vie en couple demeure dictée par les règles d'alliance (2000). Un choix autre expose à la réprobation familiale, voire aux coups, et rend difficile ensuite l'officialisation coutumière de l'union par un échange de mariage. Or l'enquête par questionnaire indique que, pour $84 \%$ des femmes kanak mariées, quels que soient leur âge et leur niveau d'éducation, il y a bien eu un tel échange qui suppose l'approbation des deux groupes de parenté.

L'inacceptable dans les relations sexuelles est défini par la non-conformité aux règles, nullement par l'absence de consentement ou l'usage de la violence. Les langues kanak, comme d'autres langues mélanésiennes - notamment en Nouvelle-Guinée (Strathern, 1985; Banks, 2000), rassemblent ainsi sous une appellation unique qui pourrait se traduire par « relations hors normes » une liaison consentie avec un(e) partenaire avec qui il est illicite de se marier ${ }^{9}$, l'adultère et le viol. La contrainte sexuelle d'une jeune fille ou d'une femme n'est réprouvée que lorsque l'auteur est en dehors des règles d'alliance, mais non quand il est défini comme « en droit » de lui imposer des relations, qu'il soit

6. Le terme ajië döwi signifie « rien », « négligeable » ou « sans valeur ». On l'utilise comme substantif pour désigner une ou des femmes tandis qu'il devient un adjectif s'il s'agit d'un homme que l'on veut disqualifier et l'on précise alors un homme de rien.

7. Lors d'une réunion publique de restitution des principaux résultats de l'enquête, une enseignante kanak, originaire de Maré, dans sa cinquantaine, prit la parole pour expliquer : « Chez nous les Kanak, la gifle c'est un signe d'amour du mari à sa femme » $(25 / 02 / 04)$.

8. Les questions concernaient une série de faits dont les gifles et les coups, les menaces avec une arme ou un objet dangereux, les séquestrations ou les expulsions du domicile, les tentatives de meurtre, et les saccages des affaires personnelles.

9. Si c'est un(e) cousin(e) parallèle, il ne s'agit plus d'une relation illicite, mais strictement interdite, c'est-à-dire un inceste, car, dans la parenté kanak, sur la Grande Terre, les germains comme les cousins parallèles sont des frères et des sœurs. 
un mari potentiel ou déjà son époux. Il existe d'ailleurs un lien tout à fait explicite entre violence et masculinité. L'on donnait autrefois à certaines massues la forme de l'organe sexuel masculin, pénis et testicules. En retour ce dernier est communément assimilé à une arme. Dans une conversation en français avec Christine Hamelin (1996), la métaphore utilisée par une jeune locutrice paicî pour désigner le sexe d'un garçon fut tout naturellement «sa guerre". L'enquête par questionnaire indique que 12,5\% des femmes kanak déclarent avoir vécu un premier rapport sexuel forcé sans qu'il n'y ait de variation significative selon les générations, ce qui établit la permanence aujourd'hui de l'entrée contrainte dans la sexualité, presque aussi fréquente chez les moins de 25 ans que chez les plus de 45 ans. Dans la mesure où l'on sait que la peur du viol conditionne les femmes bien au-delà de celles qui y sont confrontées, l'entrée contrainte dans la sexualité participe d'une socialisation des adolescentes à la soumission sexuelle ultérieure dans la sphère conjugale. Et l'enquête révèle qu'en effet $14 \%$ des femmes kanak en couple ont subi au cours des douze derniers mois des rapports sexuels contraints, où le conjoint fait usage de la force ou impose des actes sexuels non voulus. Le taux de viols conjugaux ne baisse pas de façon significative avec l'âge et demeure élevé, y compris chez les plus de 45 ans.

Chez les jeunes, tout écart d'une fille par rapport aux normes sociales, toute imprudence même, semblent pouvoir légitimer les abus sexuels masculins à son encontre. Une série d'entretiens sur les constructions de la masculinité chez les moins de 25 ans (2006) révèle la persistance de viols collectifs désignés en français local sous l'appellation "la chaîne ", une pratique nommée konvoi à Fiji (Monsell-Davis, 1986), long line aux Salomon ou line up sex en Papouasie-Nouvelle-Guinée (Jenkins, 1994 ; Borrey, 2000). L'ancienneté en NouvelleCalédonie de tels viols dont certains sont passés en cour d'assises dès les années 1960 (Salomon, 2003) parait remonter à bien plus loin, pendant l'Indigénat et même avant. Des « rapts » de femmes, c'est-à-dire des viols de guerre, sont attestés par les témoins des derniers conflits armés précédant la pacification coloniale (Thomas, 1886 ; Leenhardt, 1919) comme dans d'autres sociétés mélanésiennes - voir les Baruya (Godelier,
2003). Par ailleurs, pour humilier et punir un homme adultère, les hommes du groupe du mari offensé pouvaient violer collectivement la femme du coupable (Moncelon, 1886). Aujourd'hui, bien que les viols collectifs ne soient plus toujours légitimés par les représentations de l'ennemi ou de l'étranger, la plupart des garçons ne les considèrent pas comme répréhensibles. Soit ils sont présentés comme un châtiment " mérité » pour une fille qui a eu un comportement vestimentaire, verbal ou sexuel jugé provocant, en rupture avec la norme de soumission, soit comme un " jeu » où il s'agit de « partager » une fille entre copains ou de "profiter » d'une occasion. Toute fille ivre ou sous l'emprise du cannabis en compagnie de garçons qui ne sont pas ses frères prend le risque d'être violée, et celle qui accepte un rendez-vous avec l'un des garçons de la bande sans savoir qu'il amènera les autres, ou bien qui cède parce qu'elle craint des violences physiques supplémentaires si elle tente de s'enfuir ou de s'opposer, est supposée consentir. La jeunesse des auteurs (entre 15 et 25 ans), leur nombre qui peut aller jusqu'à dix ou quinze, leurs déclarations interrogent sur la socialisation masculine à la prédation sexuelle. Les victimes sont le plus souvent de très jeunes filles qui sont vulnérabilisées, soit par leur statut social, soit par un handicap mental, soit par la consommation de produits lors d'une fête ou par leur «mauvaise» réputation. L'opprobre qui ne manquerait pas de les accabler explique leur silence ${ }^{10}$. Une jeune femme ( 23 ans) résume ainsi la situation :

«Si ça arrive à une fille que tout le monde passe dessus c'est qu'elle a cherché, par exemple elle est saoule, elle est molasse, elle se laisse faire. Tout le monde va le savoir le lendemain mais elle, elle va se prendre pour une victime, elle va le dire ? Mais c'est elle qui a voulu [ce qui s'est passé] ! » (Extrait d'entretien, 2006)

Quand toutefois la fille ose se plaindre, et que ses parents portent l'affaire devant le conseil des anciens ${ }^{11}$, la sanction est généralement une bastonnade publique des auteurs (voir ci-dessous la description de l'affaire d'Ouvéa). Mais il se peut aussi que tous les jeunes du coin, garçons et filles, y compris la victime du viol, soient publiquement fouettés, pour rappeler l'impératif de soumission de l'ensemble des jeunes aux aînés. Dans

10. Une jeune femme kanak adulte (de plus de 18 ans) a néanmoins déclaré un viol collectif au cours des douze derniers mois lors de l'enquête par questionnaire en population générale.

11. Les conseils des anciens sont des institutions issues des remaniements liés à la présence coloniale dans des unités de résidence elles-mêmes issues du cantonnement antérieurement imposé aux Kanak. Leur autorité en matière de décision et d'application des sanctions tend à se substituer à celle des aînés de chaque groupe de parenté mais n'a jamais réussi à faire disparaître totalement cette dernière. 
le cas où le viol collectif se produit lors d'une fête dans une autre localité, il arrive que toutes les filles qui y étaient allées ainsi que leurs mères supposées les surveiller se fassent corriger, ce qui exprime alors la règle de subordination de toutes les femmes aux hommes. Quand un processus de réconciliation sociale a lieu, ce n'est pas le pardon de la victime elle-même qui compte. Les clans des violeurs viennent faire un geste d'excuse compensatoire auprès des parents ou des oncles de la fille, et éventuellement aussi auprès du lignage sur le sol duquel le viol a été perpétré et que cette sexualité en dehors des normes a souillé. Et dans la mesure où les règles d'alliance l'autorisent, les parties en présence peuvent également conclure une promesse de mariage entre la victime et celui des violeurs censé être son petit ami.

\section{Coups de trique ou années de prison ?}

Un événement médiatico-judiciaire devait fortement contribuer à bouleverser la donne - sur la construction médiatique d'un événement, voir Alban Bensa (2006 : 171-195) ${ }^{12}$. En avril 1991, la cour d'assises de Nouméa eut à se prononcer sur un viol collectif commis par dix hommes, pourtant porté l'année précédente devant le Conseil des anciens d'un village kanak d'Ouvéa. L'instigateur était l'ancien ami de la jeune femme victime, père de deux de ses enfants. Leur union contrevenant aux règles d'alliance, ses parents avaient arrangé son mariage avec un autre homme, mariage auquel elle avait cédé avant de le rompre au bout de quinze jours dans l'intention de retourner avec son ami. Entre temps, ce dernier avait entrepris une nouvelle liaison. Comme il trouvait que la mère de ses enfants avait tenu des propos insultants à son égard, pour la punir, il lui avait fait donner rendez-vous en un lieu écarté où il l'avait rouée de coups et violée avec neuf autres comparses de la localité. La victime s'était plainte auprès du conseil des anciens qui avait condamné les agresseurs à recevoir publiquement cinq coups de nerf de bœuf chacun, torse nu. La jeune femme et sa mère avaient trouvé la sanction dérisoire. Elles auraient notamment voulu qu'ils soient totalement déshabillés pour être fouettés et ainsi davantage humiliés, comme autrefois dans les châtiments coutumiers des délits sexuels où les coups visaient justement les parties génitales.
Elles étaient donc allées porter plainte à la gendarmerie et les violeurs avaient été arrêtés. En prison, ils avaient de leur côté rédigé une lettre pour récuser le tribunal, argumentant que les Mélanésiens étaient la seule ethnie en NouvelleCalédonie à être deux fois jugée. Lors du procès, tous revendiquèrent le bien-fondé de leur acte. L'avocat général requit à leur encontre de lourdes peines dont il expliqua qu'elles devaient être à la hauteur de celles infligées l'année précédente aux sept violeurs, également kanak, d'une jeune femme, elle européenne, à Saint-Louis, près de Nouméa. Les jurés le suivirent et infligèrent aux coupables cinq à douze ans de réclusion (la peine maximale étant de quinze ans).

L'institution judiciaire, déconsidérée auprès des Kanak ${ }^{13}$, trouvait une occasion d'affirmer son impartialité et de faire savoir que désormais, en Nouvelle-Calédonie, la même considération serait portée à toute victime, kanak ou européenne. Que la presse ait rapporté que le clan de la jeune femme était indépendantiste, qu'en revanche celui du principal accusé était loyaliste, et que surtout les faits se soient passés à Ouvéa où quelques années auparavant la répression organisée par l'État français contre la population avait été si violente, ne furent pas étrangers à la publicité qui entoura l'affaire. Dans le contexte du rétablissement de la paix civile et de la reconnaissance de l'identité kanak par les accords de Matignon trois ans auparavant, l'état de droit s'affirmait à nouveau comme tel, en montrant que la qualité de loyaliste ne garantissait plus l'impunité. Mais la leçon essentielle retenue par les femmes fut certainement la disparité entre les peines infligées pour un même acte par un règlement coutumier et par le tribunal.

La session d'assises qui suivit en novembre 1991 se signala par un élément lui aussi nouveau : sept sur les douze crimes à passer en jugement étaient des viols incestueux. Parmi eux, le viol à Maré d'une mineure de seize ans par ascendant adoptif, le conjoint de sa mère. Il avait fait l'objet d'une dénonciation anonyme après avoir été pourtant auparavant coutumièrement châtié par sept coups de trique. L'affaire qui ne présentait pas le même intérêt politique que celle d'Ouvéa ne fut pas du tout médiatisée bien qu'elle la rappelât dans la mesure où, à nouveau, l'écart de traitement des crimes sexuels par la coutume et la justice était démontré. Le beau-

12. Les données de ce chapitre proviennent de plusieurs sources, entretiens ethnographiques, consultation des archives du tribunal, recension de la chronique judiciaire du quotidien Les Nouvelles calédoniennes et reprennent une première version publiée par Christine Salomon dans la revue Terrain en 2003

13. En octobre 1987, le tribunal de Nouméa avait acquitté les auteurs de l'embuscade du 5 décembre 1984 qui avaient assassiné à Hienghène dix militants kanak indépendantistes. 
père incestueux fut en effet condamné à sept ans de prison, et encore la cour lui reconnut-elle des circonstances atténuantes au terme d'un débat sur le champ d'application de la coutume.

Le retentissement du procès des violeurs d'Ouvéa fut tel qu'au congrès du FLNKs qui se tenait en fin d'année, quelques leaders interpellèrent les militants indépendantistes sur les méfaits de la "chaîne ». Peu de temps après (1992), Marie-Claude Tjibaou, veuve du dirigeant kanak assassiné, devenait la première présidente de sos Violences sexuelles. L'association se spécialisa dans la dénonciation des abus sexuels les plus réprouvés socialement, ceux perpétrés contre les enfants, sans cependant jamais oser s'attaquer publiquement aux viols conjugaux. Elle s'adressait aux femmes en tant que dispensatrices de la vie et éducatrices, garantes de l'avenir de l'ensemble du groupe social, dans « leur rôle de mamans et de tantines » (MarieClaude Tjibaou, Combat ouvrier 39, mars 1995), mais pas encore comme partenaires sexuelles revendiquant leurs droits propres.

Assez rapidement toutefois, en 1999, une nouvelle association, Femmes et violences conjugales, elle aussi présidée par une Kanak, Éliane Ixeco, ouvrait un local à Nouméa pour recevoir et accompagner les femmes et faire mieux connaître les dispositions du code pénal français (en vigueur depuis 1994) pour lequel la qualité de conjoint ou de concubin de la victime constitue une circonstance aggravante des coups et blessures. Parallèlement, les associations de femmes, après avoir fait campagne contre les abus d'alcool des hommes, commençaient elles aussi à faire de la lutte contre les violences conjugales un de leurs objectifs, ce qui allait aboutir à la tenue en province Nord en mai 2002 d'un colloque sur les violences. La protestation franchissait ainsi une étape supplémentaire.

\section{La mise en question des modèles anciens}

S'ajoutant à l'action ancienne des missions et aux mesures dictées par l'administration coloniale qui avaient contribué à modifier les rapports sociaux de sexe et notamment à faire disparaître progressivement la polygynie, des changements notables avaient modifié la vie des femmes kanak dès les années 1950, après la fin du régime de l'indigénat. Parmi eux, l'octroi du droit de vote, une meilleure scolarisation, l'implication grandissante dans le monde du travail et l'urbanisation, mais aussi « la disparition progressive des formes sexualisées de domination coloniale » (Guiart, $1996: 244$ ) qui avaient longtemps lourdement pesé sur les Kanak (ainsi que sur les femmes importées comme travailleuses sous contrat des Nouvelles-Hébrides, d'Indochine et de Java). L'histoire de la colonisation pénale et libre, dans laquelle il y a eu chez les Européens un important déséquilibre du sex ratio en faveur des hommes qui ne s'est réduit significativement qu'à partir de la seconde moitié du Xx ${ }^{\mathrm{e}}$ siècle (Merle, 1995), n'avait pu en effet que renforcer au détriment des femmes kanak l'imbrication et la superposition entre violences coloniales (tout à la fois politiques, socioéconomiques et symboliques) et violences de genre. Cet agencement durable et très particulier dans lequel le déni de l'autochtonie, la relégation, parfois les tentatives d'anéantissement, le racisme, la pauvreté, le manque d'accès à l'éducation et aux soins viennent s'ajouter aux inégalités de genre, explique que les taux de violences faites aux femmes soient aujourd'hui encore nettement plus élevés chez les Kanak de NouvelleCalédonie, comme chez les Indiens et les Inuit d'Amérique, les Maoris de Nouvelle-Zélande et les Aborigènes d'Australie, que dans toutes les autres communautés de ces pays (Ferrante et al., 1996 ; Tjaden and Thoennes, 2000 ; Morris and Reilly, 2003).

La lutte nationaliste a d'emblée ouvert un nouvel espace aux femmes kanak, en leur offrant la possibilité d'intervenir en leur nom propre sur la scène politique. Dès 1975, quelques mois après l'adoption du mot d'ordre d'indépendance par l'un des groupes à l'origine du Parti de libération kanak (PALIKA), Déwé Gorodé et quelques militantes ouvraient un débat sur la place des femmes et s'organisaient pour participer à une conférence de préparation à Suva puis à la conférence mondiale des femmes à Mexico organisée par l'onu dans le cadre de l'année de la femme. Le non-respect de la décision du second congrès du PALIKA de créer une section féminine devait provoquer la création du Groupe de femmes kanak exploitées en lutte - GFKeL (Déwé Gorodé, com. pers., 2006), une organisation se définissant comme autonome, indépendantiste et féministe. Ce petit groupe féministe radical d'une soixantaine de militantes, des Kanak et quelques Européennes, pour la plupart très jeunes, se trouva vite marginalisé dans le FLNKS auquel il avait adhéré. Sa représentante au bureau politique du FLNKS se fit agresser physiquement pendant une réunion et finalement le groupe cessa ses activités en 1986 (Danielle Prébin, com. pers., 1996). De retour à la paix civile après les " événements », lors de la décennie qui suivit les accords de Matignon, les listes indépendantistes ne présentèrent aucune femme en position éligible. Le champ purement politique sem- 
blait de facto interdit aux femmes, cantonnées dans des associations féminines, les " groupes de femmes ", formées sur une base résidentielle ou d'appartenance religieuse. Ces associations s'engouffrèrent néanmoins dans la brèche ouverte par les services chargés des droits des femmes créés par l'administration française en 1989 pour promouvoir l'égalité des droits ${ }^{14}$. Les groupes de femmes ont ainsi constitué non seulement un lieu d'échanges, de mise en commun de savoirs, de partage des difficultés, mais aussi un espace social féminin collectif permettant d'échapper à la réclusion domestique par la participation à des réunions, des déplacements, des rencontres provinciales et nationales, autant d'événements rompant l'ennui et la monotonie. Souvent dévalorisé par les hommes à cause de l'effet de décentrement produit par rapport au foyer, mais moins combattu cependant par eux que l'accession des femmes à la parole politique, cet espace a été massivement investi par les femmes kanak vivant dans les zones rurales. Les données de l'enquête en population générale indiquent que c'est bien parmi les moins diplômées que ces associations mobilisent. Elles regroupent plus d'un quart des Kanak rurales. L'engouement pour les bingo, pour les sorties de groupes de femmes quel qu'en soit le motif, comme pour les feuilletons télévisés a pu d'ailleurs entraîner un véritable absentéisme domestique des jeunes femmes, à comprendre comme une forme élémentaire de résistance et le pendant d'une condition aliénée. Mais, à côté de satisfactions substitutives et d'intérêts centrés sur la valorisation des savoirs anciens comme le tressage ou la cuisine, les rassemblements de femmes ont aussi rapidement permis l'expression d'intérêts émancipateurs s'attaquant à la domination et à la subalternité, facilitant l'accès des femmes aux ressources économiques, organisant des manifestations contre les abus d'alcool, les violences physiques et sexuelles et l'utilisation de l'image des femmes par la publicité. En 1996, lors d'une rencontre organisée par le conseil national pour les droits du peuple autochtone (Nouméa), une responsable des groupes de femmes des Îles Loyauté, Denise Kacatr, déclarait :

« La femme est habituée à servir quand les hommes ont l'habitude d'être servis ou de se servir. Mais, de plus en plus, elle est appelée à prendre des responsabilités. »

C'est le débat sur la parité politique, puis son application en 2002, qui ont favorisé le retour des femmes dans la sphère politique et qui ont eu pour conséquence en Nouvelle-Calédonie de marginaliser la position des institutions coutumières qui continuent de défendre le principe d'exclusion des femmes. Chose totalement impensable il y a peu, des élues et des associations de femmes kanak se sont récemment attaquées à ce bastion sexiste qu'est le Sénat coutumier en faisant campagne pour qu'il s'ouvre aux candidatures féminines (en 2005).

La volonté de combler le fossé social qui séparait les Kanak des autres communautés en Nouvelle-Calédonie et le rééquilibrage économique entrepris dans les années 1990, malgré la persistance d'importantes inégalités, paraissent avoir accéléré les mutations dans la vie des femmes. L'on constate chez les jeunes Kanak non seulement une amélioration de l'accès à l'enseignement supérieur et au travail salarié, mais aussi à la contraception et à l'avortement légal ${ }^{15}$. Ces changements se répercutent sur les structures familiales, avec, par rapport aux générations d'avant, une diminution du nombre de naissances, une moindre stabilité des couples et un déclin de l'institution du mariage. Un autre facteur de transformation dans la dernière décennie a certainement été la généralisation de l'électrification qui permet non seulement un allégement des tâches domestiques mais aussi de suivre les séries télévisées jusque dans les zones rurales les plus reculées, ce qui contribue à promouvoir des modèles féminins dans lesquels les tâches domestiques et de reproduction ne sont plus les seules valorisées et dans lesquels le primat de la masculinité ne s'exerce qu'accessoirement par le moyen de la violence physique. Bien que la porosité entre communautés puisse paraître encore faible en Nouvelle-Calédonie, l'enquête en population générale indique qu'une femme kanak sur huit est maintenant en couple avec un homme d'une autre communauté. Dans ces unions mixtes, les femmes sont d'ailleurs tendanciellement moins exposées aux violences du conjoint, à la différence des couples mixtes en France où elles sont plus exposées (Jaspard et al., 2003).

14. La notion même de droits des femmes avait d'ailleurs soulevé tant de remous que les hommes politiques kanak qui dirigent la province Nord ont préféré l'appellation « Mission de la femme » qui, d'une part, évacue la revendication d'égalité liée à l'idée de droits et, d'autre part, véhicule une vision essentialisée du genre, l'utilisation du singulier étant un processus discursif qui renvoie à la généralité de l'espèce.

15. C'est seulement en 1995, vingt ans après la France, que la loi Veil sur l'IVG a été appliquée en Nouvelle-Calédonie et c'est seulement depuis 2006 que la pilule du lendemain est fournie aux adolescentes qui en font la demande, non seulement par les infirmières scolaires, mais aussi gratuitement dans les pharmacies. 
De façon générale, les femmes kanak justifient de moins en moins les violences physiques et sexuelles dont elles sont victimes et leur propre domination. Le taux tout à fait insignifiant d'abandon dans l'enquête par questionnaire (inférieur à $1 \%$ ) constitue un bon indicateur du refus de taire les faits. Mais l'un des principaux résultats de cette enquête réside dans la mise en évidence de changements dans les représentations de la légitimité des violences et au-delà de la conjugalité. Parmi les femmes kanak, $94 \%$ s'accordent à trouver que c'est une bonne chose qu'on parle maintenant davantage des viols. Sur cette question qui exprime le refus massif de l'occultation des violences sexuelles et de l'impunité des auteurs, il n'existe pas de différence statistiquement significative entre Européennes et Kanak.

La réprobation de la violence conjugale quelles qu'en soient les raisons et la contestation de l'autorité masculine dans la famille se généralisent également bien qu'il reste davantage de variations entre communautés. Mais l'on constate que, plus les femmes sont jeunes et ont une vie urbaine, plus ces écarts se réduisent. Pour $93 \%$ des Kanak interrogées $(96 \%$ des Européennes) il est devenu inacceptable qu'un homme frappe sa femme. L'alcool n'est pas considéré comme une circonstance atténuante par $90 \%$ des femmes kanak $(98 \%$ des Européennes), avec une forte différence d'opinion entre les rurales qui sont encore $11 \%$ à considérer que l'ivresse peut excuser les coups, alors que les urbaines ne sont que $1 \%$. Pour $96 \%$ des femmes kanak (99\% des Européennes), le refus des relations sexuelles n'apparaît pas non plus comme une justification de la violence physique du conjoint. Il reste $15 \%$ des Kanak (aucune chez les Européennes) à trouver qu'un homme a de bonnes raisons de frapper sa compagne si elle ne fait pas son travail ménager comme il faut, l'âge étant un facteur déterminant puisque les plus de 45 ans sont $24 \%$ dans ce cas alors que les moins de 25 ans sont deux fois moins nombreuses. Enfin $19 \%$ de Kanak (contre 1\% d'Européennes) excusent encore la violence d'un homme qui soupçonne sa conjointe d'infidélité, sans qu'il y ait d'écart significatif entre les âges, mais avec un score encore une fois bien plus fort chez les rurales $(21 \%)$ que chez les urbaines $(6 \%)$.

Non seulement la plupart des femmes kanak désapprouvent la violence conjugale, mais aussi les normes anciennes de soumission aux maris sont mises en cause : $90 \%$ des femmes kanak (95\% des Européennes) trouvent normal de demander des comptes au conjoint s'il rentre tard souvent ; $76 \%$ d'entre elles (94\% des Européennes) considèrent qu'une femme a le droit d'exprimer son opinion en public même si elle est en désaccord avec son mari, les jeunes et les urbaines étant davantage sur cette position que les plus âgées et les rurales. Aujourd'hui, deux tiers des femmes kanak (62\%) contestent jusqu'à l'idée que c'est à l'homme de commander dans la famille ( $86 \%$ chez les Européennes). Là encore, les moins de 25 ans sont deux fois plus nombreuses que les plus de 45 ans à refuser le bien-fondé de l'autorité masculine. Tous les résultats chiffrés traduisent une tendance à l'homogénéisation des représentations entre femmes kanak et européennes. La contestation des rôles traditionnels apparaît particulièrement forte chez les jeunes femmes kanak et celles qui vivent en ville. Ces transformations constituent un facteur important de changement ultérieur dans la mesure où la justification de leur propre soumission par les femmes contribue évidemment à la perpétuer.

Il reste certes un écart, considérable, entre le refus massif de certaines situations et le vécu des femmes au quotidien. Dans l'enquête, seules $7 \%$ parmi les Kanak qui ont déclaré des violences physiques et $2 \%$ de celles qui ont déclaré des violences sexuelles sont allées à la police ou la gendarmerie. Qui plus est, ces dénonciations ne donnent pas forcément lieu à des jugements car ensuite certaines plaintes, sous la pression, sont retirées ou bien l'affaire est classée sans suite. La faiblesse de l'utilisation des recours s'explique à la fois par l'espace insulaire où il est difficile aux femmes de se mettre vraiment à l'abri et par l'imbrication et parfois la totale superposition des réseaux familiaux et des autres réseaux sociaux, surtout dans les régions rurales. Un échappatoire pour les femmes en grande difficulté reste le départ pour la ville (Hamelin, 2000) qui exige des moyens qu'elles n'ont pas toujours et qui leur fait courir le risque d'être à la fois rejetées par leur groupe de parenté, et d'être amenées à vivre dans la précarité, souvent dans un squat, dans le Grand Nouméa.

Les femmes utilisent encore d'autres stratégies pour éviter ou réduire les violences. Elles peuvent s'éloigner pour les fuir, en retournant pour un répit dans leur propre famille. Parmi les femmes kanak habitant en couple au moment de l'enquête, $17 \%$ s'étaient séparées antérieurement de ce conjoint au moins trois mois avant de se remettre avec lui (une proportion deux fois plus importante que chez les Européennes). Les retours au foyer conjugal s'expliquent par le fait que les frères, s'ils tolèrent qu'une sœur mariée 
prolonge un peu sa visite chez eux, prennent rarement clairement parti pour elle. De plus, quitter définitivement le conjoint signifiait jusqu'il y a peu lui laisser les enfants, le divorce n'existant pas dans le droit kanak et la dissolution du mariage, une procédure qui demande l'intervention non seulement d'un magistrat français mais d'assesseurs coutumiers, donnant plutôt la garde au père. L'autodéfense et la dissuasion représentent une autre solution. Dans les couples, $15 \%$ des femmes kanak déclarent avoir frappé leur conjoint au cours des douze derniers mois ( $5 \%$ chez les Européennes). Ces violences agies sont nettement corrélées aux violences subies : $84 \%$ des femmes kanak qui ont frappé leur conjoint ont elles aussi subi de sa part au cours de la même période au moins une agression physique ou sexuelle.

\section{Conclusion}

Bien qu'évidemment les recours judiciaires et associatifs pourraient être davantage utilisés, les conduites féminines de résistance, qui vont de la fuite au rapport de force verbal et physique en face-à-face, ainsi que l'expression on ne peut plus nette du refus des violences et de leur légitimation antérieure révélée par l'enquête, témoignent d'une mise en cause profonde par les femmes kanak des violences de genre. Ce faisant elles ne s'attaquent pas seulement aux effets « les plus négatifs » de la domination masculine comme les décrit Bourdieu (1998:122), mais elles sapent aussi l'un des fondements de sa reproduction, dans la mesure où la menace des violences a pour effet de conditionner l'ensemble des femmes, au-delà de celles qui les subissent directement (Tabet, 2001). On ne saurait donc parler de partage des représentations du genre entre les hommes et les femmes en NouvelleCalédonie aujourd'hui ni d'adhésion quelconque, même extorquée aux femmes, à leur propre soumission ( $c f$. la critique des concepts du consentement des femmes ou de leur adhésion formulée in Mathieu, 1999).

L'abaissement du seuil des violences tolérées par les femmes et la place qu'elles accordent à la notion de consentement sexuel distinguent désormais la Nouvelle-Calédonie des autres pays insulaires du Pacifique où ont eu lieu des enquêtes comparables, en particulier Samoa (SFHSS, 2003) où une forte proportion de femmes continue de légitimer les violences conjugales et où $70 \%$ des répondantes trouvent qu'une femme ne peut se refuser sexuellement à son mari. Les réponses à l'échelle d'acceptabilité des violences et les écarts entre les jeunes femmes kanak et leurs aînées de ce point de vue sont très révélateurs d'une évolution rapide des normes qu'il faut rapporter non seulement à un meilleur accès à l'éducation, à l'information et à l'emploi ces quinze dernières années, mais aussi à la singularité du contexte politique calédonien. La dénonciation par les jeunes nationalistes du colonialisme français et de la marginalisation massive des Kanak est apparue à la fin des années 1970, au moment même où le mouvement des femmes en Occident était à son apogée, ce qui a favorisé l'apparition d'une revendication féministe radicale portée par des jeunes femmes kanak au sein du mouvement indépendantiste. Bien que la structuration politique de cette revendication ait été éphémère et qu'elle ait été rapidement étouffée en tant que telle, elle s'est ensuite redéployée à la faveur des arrangements passés avec l'État français pour réduire les inégalités économiques et sociales. Ainsi la contestation avant-gardiste des années 1980 a-t-elle de facto ouvert la voie les années suivantes à un mouvement féministe plus réformiste, mais avec un caractère de masse et une solide implantation en milieu rural. Plus récemment, de nouvelles avancées ont été permises par l'émergence des notions de destin commun et de citoyenneté calédonienne qui accompagnent la reconnaissance de l'identité autochtone. Cette configuration très originale a constitué un terrain propice au développement et à la diversification d'un mouvement associatif féminin qui a relayé la dénonciation des violences de genre et la revendication d'égalité des droits, maintenant portées au niveau politique, depuis l'application de la parité par les femmes, aussi bien kanak qu'européennes. La féminisation du gouvernement dont la présidence a été occupée de 2004 à 2007 par une Calédonienne européenne et la vice-présidence par une militante kanak, engagée depuis le départ dans la lutte pour l'égalité, ne peut que contribuer à la tendance actuelle à l'homogénéisation des représentations du genre chez les femmes des différentes communautés.

\section{Remerciements}

L'enquête Santé et conditions de vie des femmes calédoniennes a bénéficié du concours financier de l'Agence nationale de recherches sur le sida, d'Ensemble contre le sida, de l'Institut de recherches scientifiques sur les boissons et de la Mission interministérielle de lutte contre la drogue et la toxicomanie. Le gouvernement et les provinces de Nouvelle-Calédonie l'ont aussi 
soutenue financièrement. Nous remercions Déwé Gorodé ainsi que les femmes qui se sont impliquées à nos côtés et nous dédions cet article à Éliane Ixeco, militante résolue des droits des femmes, décédée en 2006.

\section{BIBLIOGRAPHIE}

Banks Cyndi, 2000. Contextualising sexual violence: rape and carnal knowledge in Papua New Guinea, in S. Dinnen and A. Ley (eds), Reflections on Violence in Melanesia, Canberra, Hawkins Press and Asia Pacific Press, pp. 83-104.

Bensa Alban, 2006. La fin de l'exotisme. Essais d'anthropologie critique, Toulouse, Anacharsis.

Borrey Anou, 2000. Sexual Violence in Perspective: The Case of Papua New Guinea, in S. Dinnen and A. Ley (eds) Reflections on Violence in Melanesia, Canberra, Hawkins Press and Asia Pacific Press, pp. 105-18.

BOURDIEU Pierre, 1998. La domination masculine, Paris, Seuil.

BourgoIs Philippe, 2001. En quête de respect. Le crack à New York, Paris, Seuil.

_, 2002. La violence en temps de guerre et en temps de paix. Leçons de l'après-guerre froide : l'exemple du Salvador, Culture \& Conflits 47, 3, pp. 81-116.

D'ANDrade Roy, 1995. Moral Models in Anthropology, Current Anthropology 36, 3, pp. 399-408.

FARMER Paul, 1997. On Suffering and Structural Violence: A View From Below, in A. Kleinman, V. Das and M. Lock (eds.), Social Suffering, University of California Press, Los Angeles-London, pp. 261283.

Ferrante Anna, Frank Morgan, David InDERMaur and Richard HARDING, 1996. Measuring the Extent of Domestic Violence, Sydney, Hawkins Press.

GODELIER Maurice, 2003. What is a sexual act?, Anthropological Theory 3, 2, pp. 179-198.

GorodÉ Déwé, 2005. L'Épave. Nouméa, Madrépores.

Guiart Jean, 1996. Nouméa, cité métisse ?, Journal de la Société des Océanistes 103, 2, pp. 231-273.

Hamelin Christine, 2000. "Les gens de Nouméa »: mutations et permanences en milieu urbain, in A. Bensa \& I. Leblic (éds), En pays kanak. Ethnologie, linguistique, archéologie, histoire de la NouvelleCalédonie, Paris, éd. de la maison des sciences de l'homme, Mission du patrimoine ethnologique, cahier 14, pp. 339-354.

Hamelin Christine et Christine Salomon, 2004. Parenté et violences faites aux femmes en NouvelleCalédonie. Un éclairage sur l'ethnicité différentiée des violences dans la famille, Espace, Populations et sociétés 2, pp. 307-323.

JASPARD Maryse, Elisabeth Brown et al., 2003. Les violences envers les femmes en France. Une enquête nationale, Paris, La Documentation française.
Jenkins Carol, 1994, National study of sexual and reproductive knowledge and behaviour in Papua New Guinea, Goroka, PNG Institute of Medical research monograph 10.

LeBLIC Isabelle, 2000. Le dualisme matrimonial paicî en question (Ponérihouen, Nouvelle-Calédonie), L'Homme 154-155, pp. 183-204.

LeENHARDT Maurice, 1907. Lettre au Journal des Missions, Journal des missions évangéliques.

-, 1919. Rapport de la Mission de Nouvelle-Calédonie et Maré pour 1918, Journal des missions évangéliques.

MathiEu Nicole-Claude, 1999. Bourdieu ou le pouvoir auto-hypnotique de la domination masculine, Les Temps modernes 604, pp. 286-324.

MerLe Isabelle, 1995. Expériences coloniales: La Nouvelle-Calédonie, 1853-1920, Paris, Belin.

MonCelon Léon, 1886, Réponse alinéa par alinéa, pour les Néo-Calédoniens au Questionnaire de sociologie et d'ethnographie de la Société, Bulletin de la société d'anthropologie de Paris, $3^{\mathrm{e}}$ série, tome 9, pp. 345-380.

Monsell-Davis Michael, 1986, It's a man's game: identity, social role, social change and delinquency in Suva, in C. Griffin and M. Monsell-Davis (eds), Fijians in Town, Suva, usP, Institute of Pacific Studies, pp. 132-165.

MorRIS Allison and James ReILLY (in collaboration with Sheila BERRY and Robin RANSOM), 2003. New Zealand National Survey of Crime Victims 2001, Wellington, Ministry of Justice.

SALOMON Christine, 1998. La personne et le genre au centre-nord de la Grande Terre (NouvelleCalédonie), Gradhiva 23, pp. 81-100.

-, 2000a. Les femmes kanak face aux violences sexuelles et domestiques : le tournant judiciaire des années 1990, Le Journal des anthropologues 82, pp. 287-307.

—, 2000b. Hommes et Femmes : harmonie d'ensemble ou antagonisme sourd?, in A. Bensa \& I. Leblic (éds), En pays kanak. Ethnologie, linguistique, archéologie, histoire de la Nouvelle-Calédonie, Paris, éd. de la maison des sciences de l'homme, Mission du patrimoine ethnologique, cahier 14, pp. 311-338.

—, 2002. A'jië and Paicî Kanak Societies, a Women's Perspective: Obligatory Maternity and Reproductive Autonomy, in M. Jolly \& V. Lukere (eds), Birthing in The Pacific: Beyond Tradition and Modernity, Honolulu, University of Hawai'i Press, pp. 7999.

_, 2003. Quand les filles ne se taisent plus : un aspect du tournant post-colonial en Nouvelle-Calédonie, Terrain 40, pp. 133-150.

SCHEPER-Hughes Nancy, 1996. Small wars and invisible genocides, Social Science \& Medicine 43, 5, pp. 889-900.

STRATHERn Marilyn, 1985. Introduction, in S. Toft (ed), Domestic Violence in Papua New Guinea, Port 
Moresby, Law Reform Commission of Papua New Guinea, Monograph 3, pp. 1-13.

TABET Paola, 2001. La grande arnaque. L'expropriation de la sexualité des femmes, Actuel Marx 30, pp. 131-152.

SpC-UnfPA, 2003. The Samoa Family Health and Safety Study, Nouméa, Graphoprint.
Thomas Julian («The Vagabond»), 1886. Cannibals and Convicts. Notes of personal experiences in the Western Pacific, London, Cassell \& Company.

TJADEN Patricia and Nancy Thoennes, 2000. Extent, Nature, and Consequences of Rape Victimization: Findings From the National Violence Against Women Survey, NIJ Special Report, www.ojp.usdoj.gov/nij. 